\title{
Comparative safety of direct oral anticoagulants and warfarin in venous thromboembolism: multicentre, population based, observational study
}

\begin{abstract}
Min Jun, ${ }^{1,2,3}$ Lisa M Lix, ${ }^{4}$ Madeleine Durand, ${ }^{5}$ Matt Dahl, ${ }^{6}$ J Michael Paterson,,${ }^{7,8,9}$ Colin R Dormuth, ${ }^{10}$ Pierre Ernst, ${ }^{11,12}$ Shenzhen Yao, ${ }^{13}$ Christel Renoux, ${ }^{11,14,15}$ Hala Tamim, ${ }^{16,17}$ Cynthia Wu, ${ }^{18}$ Salaheddin M Mahmud, ${ }^{19}$ Brenda R Hemmelgarn, ${ }^{1}$ for the Canadian Network for Observational Drug Effect Studies (CNODES) Investigators
\end{abstract}

\begin{tabular}{l}
\hline For numbered affiliations see \\
end of article. \\
Correspondence to: \\
B Hemmelgarn \\
Brenda.Hemmelgarn@ahs.ca \\
Additional material is published \\
online only. To view please visit \\
the journal online. \\
Cite this as: BMJ 2017;359:j4323 \\
http://dx.doi.org/10.1136/bmj.j4323
\end{tabular}

Accepted: 11 September 2017

\section{ABSTRACT}

OBJECTIVE

To determine the safety of direct oral anticoagulant (DOAC) use compared with warfarin use for the treatment of venous thromboembolism.

DESIGN

Retrospective matched cohort study conducted between 1 January 2009 and 31 March 2016.

\section{SETTING}

Community based, using healthcare data from six jurisdictions in Canada and the United States.

\section{PARTICIPANTS}

59525 adults (12 489 DOAC users; 47036

warfarin users) with a new diagnosis of venous thromboembolism and a prescription for a DOAC or warfarin within 30 days of diagnosis.

\section{MAIN OUTCOME MEASURES}

Outcomes included hospital admission or emergency department visit for major bleeding and all cause mortality within 90 days after starting treatment. Propensity score matching and shared frailty models were used to estimate adjusted hazard ratios of the outcomes comparing DOACs with warfarin. Analyses were conducted independently at each site, with meta-analytical methods used to estimate pooled hazard ratios across sites.

RESULTS

Of the 59525 participants, 1967 (3.3\%) had a major bleed and 1029 (1.7\%) died over a mean follow-up of 85.2 days. The risk of major bleeding was similar for DOAC compared with warfarin use (pooled hazard ratio $0.92,95 \%$ confidence interval 0.82 to 1.03 ), with the overall direction of the association favouring

\section{WHAT IS ALREADY KNOWN ON THIS TOPIC}

Non-inferiority randomised trials have shown similar efficacy and a reduced or similar risk of major bleeding complications for direct oral anticoagulants (DOACs) compared with warfarin in the treatment of venous thromboembolism Clinical trials include a highly selected patient group, so the rate of safety events such as major bleeding reported in trials often does not reflect those observed in routine clinical practice

\section{WHAT THIS STUDY ADDS}

This large multicentre, population based study suggests that DOAC use is not associated with higher major bleeding risk in venous thromboembolism A series of sensitivity and subgroup analyses ensured the robustness of study findings and showed consistent findings in different patient groups
DOAC use. No difference was found in the risk of death (pooled hazard ratio $0.99,0.84$ to 1.16 ) for DOACs compared with warfarin use. There was no evidence of heterogeneity across centres, between patients with and without chronic kidney disease, across age groups, or between male and female patients.

CONCLUSIONS

In this analysis of adults with incident venous thromboembolism, treatment with DOACs, compared with warfarin, was not associated with an increased risk of major bleeding or all cause mortality in the first 90 days of treatment.

TRIAL REGISTRATION

Clinical trials NCT02833987.

\section{Introduction}

Venous thromboembolism, a condition that includes deep venous thrombosis and pulmonary embolism, is common with an annual incidence of approximately one case per 1000 people. ${ }^{1}$ As the third most common cause of vascular related death after myocardial infarction and stroke, venous thromboembolism is associated with considerable morbidity and premature mortality. ${ }^{2}$

Warfarin has been the primary oral anticoagulant used for treatment of venous thromboembolism but has inherent limitations that detract from its therapeutic utility, with a narrow therapeutic index and variability in patients' responses dependent on a range of factors including diet and concomitant drugs. ${ }^{3}$ In contrast, direct oral anticoagulants (DOACs) have relatively stable pharmacokinetics that remove the need for regular monitoring and dose adjustment. ${ }^{4}$ Recent non-inferiority trials assessing the efficacy and safety of DOACs compared with warfarin in the treatment of acute venous thromboembolism and prevention of recurrent venous thromboembolism have shown comparable efficacy without significantly increased risk of major bleeding. ${ }^{5-8}$ However, trials are not typically designed to detect differences in safety outcomes. In addition, bleeding rates are underestimated in randomised trials, ${ }^{9}$ as patients with a history of bleeding are usually excluded. Population based comparative assessments of the safety of DOACs for treatment of venous thromboembolism are lacking. With DOACs being increasingly used in clinical practice, a need exists to better delineate their safety using real world, population based data sources to inform clinical practice. 
We used a multicentre, population based, propensity score matched cohort of adults with incident venous thromboembolism to assess the safety (major bleeding and all cause mortality) of DOACs (dabigatran, apixaban, or rivaroxaban) compared with warfarin for the treatment of incident venous thromboembolism.

\section{Methods}

\section{Study design and source population}

We conducted a retrospective, propensity score matched cohort study using healthcare data from six jurisdictions (the Canadian provinces of Alberta, Manitoba, Ontario, Quebec, and Saskatchewan and the United States (MarketScan)) according to a prespecified common protocol (https://clinicaltrials. gov/ct2/show/NCT02833987).

\section{Cohort definition}

The study cohort included adults aged 18 years or over (66 years or over in Ontario) newly dispensed either a DOAC (apixaban, dabigatran, or rivaroxaban) or warfarin between 1 January 2009 and 180 days before the end date of the available database at each site (the latest end date was 31 March 2016). Patients were eligible for inclusion if they had a new diagnosis of venous thromboembolism, defined as at least one diagnostic code for venous thromboembolism within 30 days before the date of the first prescription for a DOAC or warfarin (the diagnosis ascertainment period). Our definition for venous thromboembolism included deep venous thrombosis and pulmonary embolism, ${ }^{10-12}$ and we identified venous thromboembolism events by using hospital admission, emergency department, and/or physician claims databases. ICD (international classification of diseases) diagnosis codes from the 9th (ICD-9) and 10th (ICD-10-CA) revisions are shown in supplementary table A. We excluded patients who had a previous diagnosis of venous thromboembolism or atrial fibrillation 335 days or less before this first venous thromboembolism diagnosis, less than one year of information in the database before the date of the incident venous thromboembolism diagnosis, a prescription for a DOAC or warfarin within one year before the cohort entry date, or both a DOAC and warfarin prescribed on the date of their first prescription.

We defined cohort entry as the date of the first prescription for a DOAC or warfarin. We matched up to five warfarin users with each DOAC user. A warfarin user could serve as a match for more than one DOAC user, but only once. Matching was based on age at cohort entry (within one year either side), sex, date of cohort entry (within one year either side), and a propensity score reflecting the probability of being treated with a DOAC at the time of cohort entry (detailed below). We considered exposure to a DOAC or warfarin as a time fixed variable throughout the study follow-up.

\section{Outcome definition}

The primary outcome was time to an incident major bleed (see supplementary table A for a list of the validated ICD-9 and ICD-10-CA diagnosis codes), defined as first hospital admission or emergency department visit for intracranial, gastrointestinal, or other bleeding in the 90 days after cohort entry. This algorithm for major bleeding has $94 \%$ sensitivity and $83 \%$ specificity. ${ }^{1314}$ The secondary outcome was all cause mortality in the 90 days after cohort entry. We followed participants from cohort entry until the earliest of the major bleeding event, death, outmigration (out of the province for Canadian sites), or study end (defined as 90 days after the cohort entry for the primary analysis). We were unable to ascertain the outcome of mortality in the US MarketScan database, as this information was unavailable.

\section{Covariates}

Information on demographic characteristics and comorbid conditions came from the healthcare data files of the included sites. We defined comorbid conditions a priori on the basis of previous research, ${ }^{15}$ and we defined clinical expertise by inpatient or outpatient ICD-9 and ICD-10-CA diagnosis codes based on information available for the 365 days before cohort entry. Comorbid conditions included diabetes, hypertension, chronic kidney disease, acute kidney injury, history of cardiovascular disease (including ischaemic heart disease, congestive heart failure, cardiomyopathy, valvular heart disease, and pacemaker or defibrillator), peripheral vascular disease, cerebrovascular disease (including transient ischaemic attack and all stroke subtypes), chronic obstructive pulmonary disease, liver disease, and cancer. We also included history of major bleeding (hospital admission or emergency department visit in the previous 365 days) and residence in long term care (for sites that could identify this). Antiplatelet agent, non-steroidal anti-inflammatory drug, or gastroprotective drug use, defined as at least one dispensed prescription within 90 days before cohort entry, came from prescription drug records.

\section{Statistical analysis}

We developed a multivariable logistic regression model that included demographic information, comorbid conditions (listed above), and prescription antiplatelet agents, non-steroidal anti-inflammatory drugs, and gastroprotective drugs to estimate the probability (propensity) of being treated with a DOAC, conditional on the included covariates. We excluded $5 \%$ of patients at each end of the propensity score distribution. We matched up to five warfarin users with each DOAC user (with replacement) on age at cohort entry date, sex, cohort entry date, and the propensity score (based on a calliper width of 0.02 of the log odds of the propensity score). We assessed the balance in the covariates between the DOAC and warfarin exposure groups before and after matching by using the standardised mean difference (expressed as a percentage), whereby an absolute standardised mean difference below 10\% implied acceptable balance. $^{16}$ 
We fitted a shared frailty model based on the matched cohort to estimate hazard ratios of study outcomes (and their 95\% confidence intervals) in the 90 days after cohort entry among patients dispensed prescriptions for DOACs, using warfarin as the reference treatment. This model accounts for the clustering of individuals in the matching process and is akin to a random effects survival model. ${ }^{17}$ We constructed separate models to assess the risk of major bleeding and all cause mortality associated with DOAC use compared with warfarin use. Analyses were conducted independently at each study site using linked, encoded data and SAS software.

\section{Secondary analyses}

In secondary analyses, we assessed the risk of major bleeding associated with DOACs compared with warfarin for the treatment of incident venous thromboembolism according to chronic kidney disease status (defined on the basis of on a validated algorithm, using ICD-9 (403 and 585) and ICD-10-CA (E102, E112, E132, E142, I12, I13, N08, N18, and N19) codes). ${ }^{18-20}$ We also assessed the risk of major bleeding and all cause mortality outcomes associated with DOAC use compared with warfarin use stratified by age group ( $\leq 65,66-75,76-85$, and $>85$ years) and sex.

\section{Sensitivity analyses}

We did several sensitivity analyses to confirm the robustness of our findings. We repeated all analyses using a venous thromboembolism diagnosis ascertainment period of 15 days, excluding all patients with a diagnosis of venous thromboembolism or atrial fibrillation during a period of 1065 days before the new venous thromboembolism diagnosis, and using an extended duration of follow-up (180 days).

\section{Meta-analysis}

Analyses were conducted independently at each participating site according to a common analytical protocol and then pooled using meta-analysis techniques with a fixed effects model, which assumes that variation between studies is the estimate associated with sampling error. We calculated inverse variance weighted pooled risk estimates and 95\% confidence intervals. We used Review Manager software, version 5.3, for analyses.

\section{Exclusion of Clinical Practice Research Database and province of Nova Scotia}

As specified in the study protocol, we had sought to include data from the UK based Clinical Practice Research Database (CPRD). However, the results on mortality obtained in the CPRD were clearly different from those at other sites. On review of the literature on venous thromboembolism and mortality using the $C P R D,{ }^{21}$ we determined that mortality from venous thromboembolism based on CPRD data are substantially underestimated using the general practice electronic records. ${ }^{22}$ We therefore excluded CPRD data. We also sought to include data from the Canadian province of Nova Scotia. However, owing to the small size of the matched cohort $(n=60)$, the limited number of major bleeding events observed across the DOAC and warfarin exposure groups (none observed in the DOAC group), and the subsequent non-convergence of models and limited ability to do additional secondary and sensitivity analyses, we excluded Nova Scotia from all analyses.

\section{Patient involvement}

This study involved the secondary use of existing data sources and did not include patients as study participants. No patients were involved in setting the research question, the study design, or the overall conduct of the study. There are no plans to involve patients in the dissemination of study findings.

\section{Results}

\section{Patients' characteristics}

We identified 148972 adults aged 18 years or over with a new prescription for a DOAC $(n=13813$; 9.3\%) or warfarin ( $\mathrm{n}=135159 ; 90.7 \%)$ and an incident diagnosis of venous thromboembolism within 30 days before this prescription (fig 1). Of these, we included 59525 adults in the matched analyses (12 489 (21.0\%) DOAC users; 47036 (79.0\%) warfarin users). Overall, after matching, balance was achieved across all included covariates (supplementary table B). The mean age of the matched cohort $(n=59525)$ was 64.3 (SD 15.9) years, and 53.6\% were female (table 1). Among DOAC users, most prescriptions were dispensed between 2013 and 2015 (88.8\%) and rivaroxaban was the most common DOAC used overall (94.9\%). The proportion of patients who switched from DOACs to warfarin or from warfarin to DOACs during the followup period was small (374 (3.0\%) and 2476 (5.3\%), respectively).

\section{Incidence rates of major bleeding and all cause mortality}

Over a mean follow-up of 85.2 days, 1029 (1.7\%) deaths occurred and 1967 (3.3\%) patients had a major bleed, of which 131 (6.7\%), 758 (38.7\%), and 1070 (54.6\%) were due to an intracranial, gastrointestinal, and other bleed, respectively. Bleeding rates at 30 days ranged between $0.2 \%$ and $2.9 \%$ for DOACs and $0.2 \%$ and $2.9 \%$ for warfarin. Bleeding rates at 60 days ranged between $0.4 \%$ and $4.3 \%$ for DOACs and $0.4 \%$ and $4.3 \%$ for warfarin.

\section{Hazard ratios for major bleeding and all cause mortality}

Overall, the risk of major bleeding associated with DOAC use among patients with incident venous thromboembolism was similar to that with warfarin use (hazard ratio 0.92, 95\% confidence interval 0.82 to 1.03; fig 2), with the direction of the association favouring DOACs. We observed no evidence of heterogeneity across included sites $\left(\mathrm{I}^{2}=1 \%, \mathrm{P}\right.$ for heterogeneity=0.41). We observed similar results for the outcome of all cause mortality (hazard ratio 0.99 , 0.84 to 1.16 ; $\mathrm{I}^{2}=0 \%$; P for heterogeneity=0.66; fig 3). 


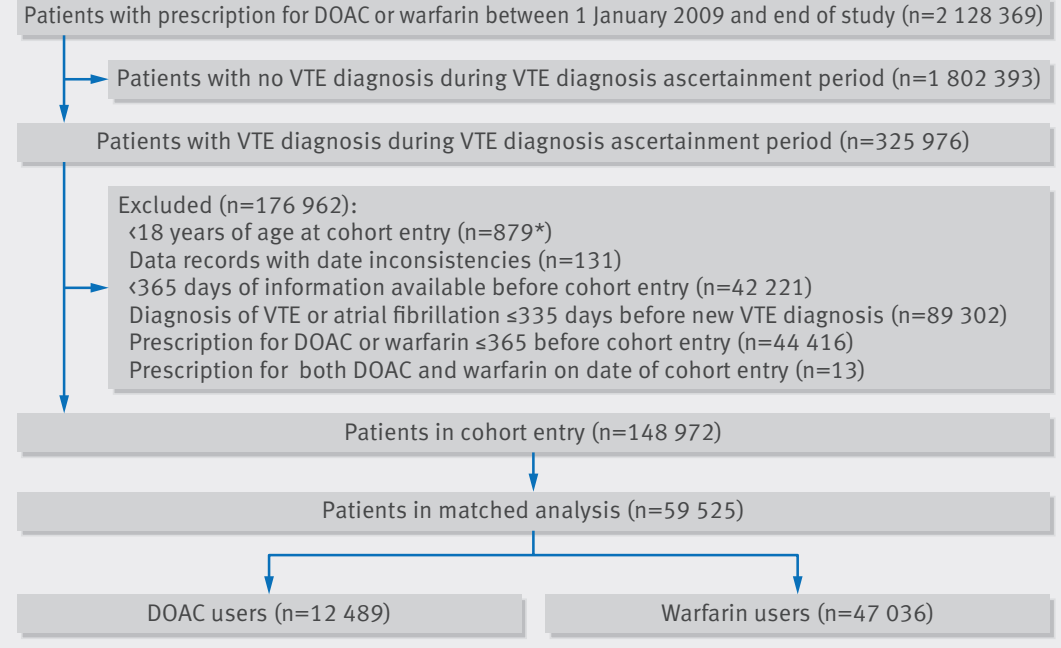

Fig 1 | Flow chart for defining study cohort. Cells of tables with patient counts $<5$ were suppressed (S) by participating sites owing to privacy restrictions. The sum of count data may thus differ slightly from the presented total

Hazard ratios for major bleeding and all cause mortality according to chronic kidney disease status, age groups, and sex

Wefound no significant heterogeneity in therisk of major bleeding and all cause mortality associated with DOAC use when we assessed patients according to chronic kidney disease status (fig 4), age (supplementary figures A and B), and sex (supplementary figure C).

\section{Sensitivity analysis}

Overall results remained unchanged in all sensitivity analyses, including when a longer period of follow-up (180 days) was used (supplementary figures D and E).

\section{Discussion}

In our multicentre, population based, propensity score matched cohort study of 59525 adults with incident venous thromboembolism, we observed that treatment with DOACs compared with warfarin was not associated with an increased risk of major bleeding or all cause mortality in the first 90 days of treatment. We found similar results for patients with and without chronic kidney disease, across all age groups, and for men and women.

Implications and comparison with other studies Randomised trials assessing the efficacy and safety of DOAC use compared with vitamin K antagonists for the treatment of venous thromboembolism have shown favourable risk-benefit profiles for DOAC treatment. ${ }^{23}$ Although we did not observe a lower risk of major bleeding with DOAC treatment in our study, given

\begin{tabular}{|c|c|c|c|c|}
\hline \multirow[b]{2}{*}{ Characteristics } & \multicolumn{2}{|l|}{ Matched cohort } & \multicolumn{2}{|l|}{ Unmatched cohort } \\
\hline & DOACs $(n=12489)$ & Warfarin $(n=47036)$ & DOACs $(n=13813)$ & Warfarin $(n=135159)$ \\
\hline Mean (SD) age, years† & $62.8(13.8)$ & $64.7(13.3)$ & $62.9(14.1)$ & $62.1(14.8)$ \\
\hline \multicolumn{5}{|l|}{ Age group (years): } \\
\hline $18-24$ & $222(1.8)$ & $680(1.4)$ & $254(1.8)$ & $2459(1.8)$ \\
\hline $25-44$ & $1573(12.6)$ & $5057(10.8)$ & $1732(12.5)$ & $19155(14.2)$ \\
\hline $45-64$ & $4508(36.1)$ & $14673(31.2)$ & $4956(35.9)$ & $51477(38.1)$ \\
\hline $65-74$ & $2951(23.6)$ & $13045(27.7)$ & $3263(23.6)$ & $26280(19.4)$ \\
\hline $75-84$ & $2274(18.2)$ & $9866(21.0)$ & $2499(18.1)$ & $25060(18.5)$ \\
\hline$\geq 85$ & $896(7.2)$ & $3715(7.9)$ & $1076(7.8)$ & $10728(7.9)$ \\
\hline Female sex & $6667(53.4)$ & $25231(53.6)$ & $7255(52.5)$ & $69135(51.2)$ \\
\hline \multicolumn{5}{|l|}{ DOAC agent: } \\
\hline Apixaban & $104(0.8)$ & NA & $112(0.8)$ & NA \\
\hline Dabigatran & $539(4.3)$ & NA & $590(4.3)$ & NA \\
\hline Rivaroxaban & $11812(94.6)$ & NA & $13071(94.6)$ & NA \\
\hline Acute kidney injury & $482(3.9)$ & $2017(4.3)$ & $632(4.6)$ & $9664(7.2)$ \\
\hline Chronic kidney disease & $370(3.0)$ & $1553(3.3)$ & $625(4.5)$ & $11175(8.3)$ \\
\hline Diabetes & $2445(19.6)$ & $8352(17.8)$ & $2727(19.7)$ & $29142(21.6)$ \\
\hline Hypertension & $5325(42.6)$ & $19273(41.0)$ & $6102(44.2)$ & $64851(48.0)$ \\
\hline Previous health services encounter for any major bleeding $\ddagger$ & $582(4.7)$ & $2255(4.8)$ & $665(4.8)$ & $8455(6.3)$ \\
\hline Cardiovascular disease & $3525(28.2)$ & $12281(26.1)$ & $4170(30.2)$ & $47079(34.8)$ \\
\hline Peripheral vascular disease & $745(6.0)$ & $2584(5.5)$ & $851(6.2)$ & $11189(8.3)$ \\
\hline Cerebrovascular disease & $618(4.9)$ & $2043(4.3)$ & $688(5.0)$ & $8777(6.5)$ \\
\hline Chronic obstructive pulmonary disease & $1570(12.6)$ & $5539(11.8)$ & $1740(12.6)$ & $20461(15.1)$ \\
\hline Liver disease & $318(2.5)$ & $1203(2.6)$ & $409(3.0)$ & $4586(3.4)$ \\
\hline Cancer & $1812(14.5)$ & $6708(14.3)$ & $1977(14.3)$ & $23518(17.4)$ \\
\hline Residence in long term care/personal care facility & $222(1.8)$ & $922(2.0)$ & $255(1.8)$ & $2214(1.6)$ \\
\hline \multicolumn{5}{|l|}{ Prescription drug use: } \\
\hline Antiplatelet drug & $2950(23.6)$ & $10012(21.3)$ & $3156(22.8)$ & $38652(28.6)$ \\
\hline Non-steroidal anti-inflammatory drug & $4471(35.8)$ & $14029(29.8)$ & $5318(38.5)$ & $56970(42.2)$ \\
\hline Gastroprotective drug & $4214(33.7)$ & $15885(33.8)$ & $4641(33.6)$ & $46156(34.1)$ \\
\hline
\end{tabular}

$\mathrm{DOAC}=$ direct oral anticoagulant; $\mathrm{NA}=$ not applicable; $\mathrm{VTE}=$ =venous thromboembolism.

${ }^{*}$ Cells of tables with patient counts $<5$ were suppressed by participating sites owing to privacy restrictions; the sum of count data may thus differ slightly from the presented total.

tMean sample size weighted age.

₹Major bleeding defined as first hospital admission or emergency department visit for intracranial, gastrointestinal, or other bleeding. 


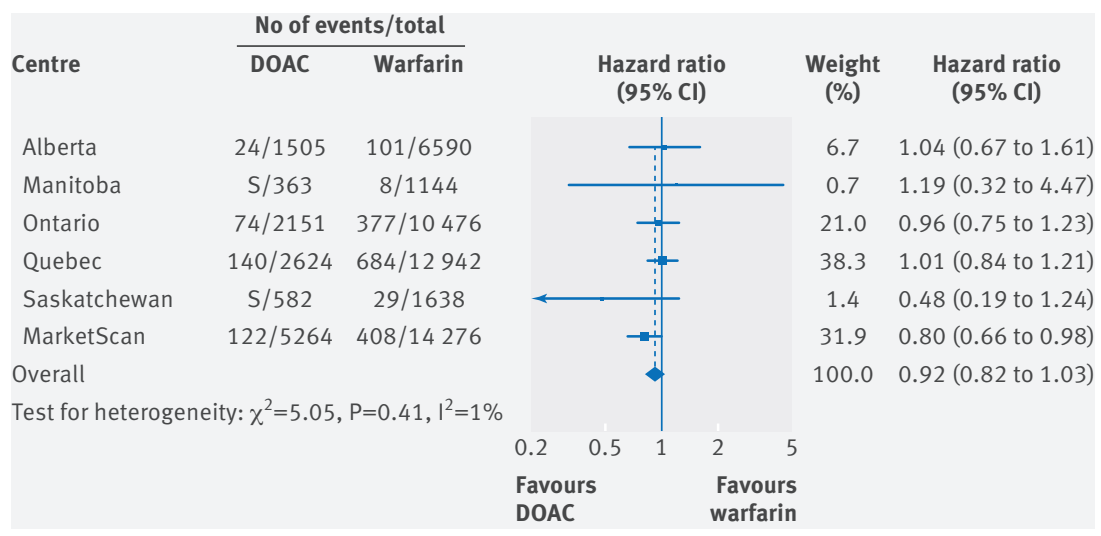

Fig 2 | Hazard ratios (95\% Cls) of major bleeding associated with direct oral anticoagulant (DOAC) use compared with warfarin use (reference category was warfarin users). $S=$ events were $<5$ and cells were suppressed

the absence of an increased bleeding risk and the advantages associated with its use particularly around frequency of monitoring and dosing, our results suggest that DOACs may be considered as a treatment option for patients with venous thromboembolism who are candidates for anticoagulation.

A recent systematic review assessing the efficacy and safety of DOACs for the treatment of acute venous thromboembolism based on data from nine trials (28554 participants and 228 major bleeding events) showed that bleeding risks were either similar (hazard ratio for dabigatran versus warfarin 0.76, 95\% confidence interval 0.48 to 1.18 ) or reduced (hazard ratio for apixaban and rivaroxaban versus warfarin 0.33 (0.18 to 0.56 ) and 0.55 (0.37 to 0.80$)$, respectively) for DOAC use compared with warfarin. ${ }^{24}$ The study also reported no differences in the risk of all cause mortality with DOAC use. Large studies on the assessment of the safety of DOAC for the treatment of venous thromboembolism in routine clinical practice have been limited. Several relatively small cohort studies of rivaroxaban have reported similarly favourable safety profiles associated with its use in patients with venous thromboembolism. ${ }^{925-29}$ In addition, a recent cohort study of 12318 venous thromboembolism patients comparing 5411 rivaroxaban users with 6907

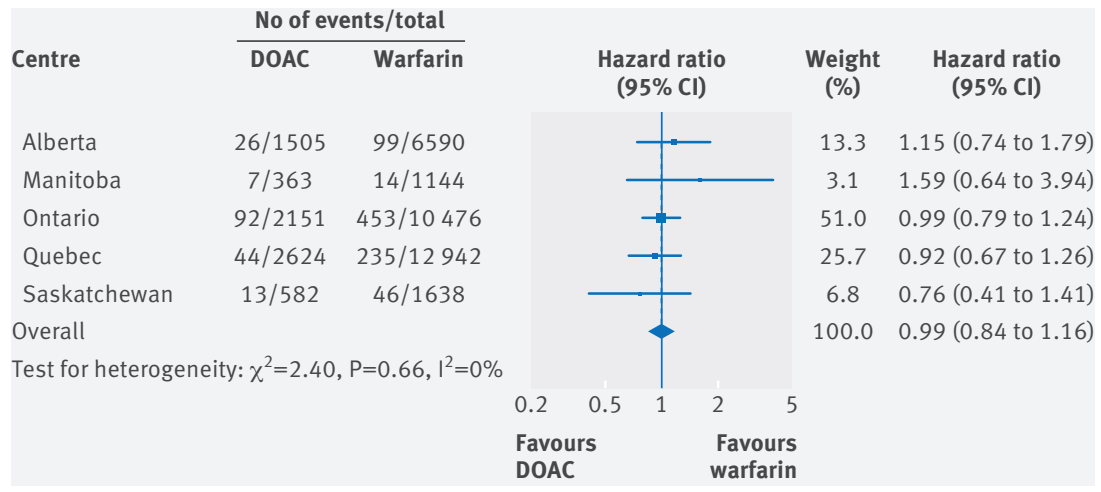

Fig 3 | Hazard ratios $(95 \% \mathrm{Cls})$ of all cause mortality associated with direct oral anticoagulant (DOAC) use compared with warfarin use (reference category was warfarin users) vitamin $\mathrm{K}$ antagonist users showed no difference in major bleeding risk (hazard ratio 1.08, 0.84 to 1.39). However, these studies are limited by their small sample size ( $\mathrm{n}=361$ to 4768 patients $^{25} 27283031$ ), lack of a comparator, ${ }^{26} 29$ and inclusion of cases of major bleeding in patients admitted to hospital only, which probably led to an underestimation of the bleeding rates. ${ }^{32}$ Our study, using a large population based cohort of DOAC and warfarin users in whom major bleeding was identified across in-hospital and emergency department data sources, has thus important implications for the management of patients with venous thromboembolism at risk of having a recurrent event.

However, DOAC use has also been associated with increased risk of bleeding in relation to specific subtypes of bleeding episodes, including gastrointestinal bleeding, in other patient populations including those with atrial fibrillation. ${ }^{33-35}$ A systematic review of 43 trials including patients with venous thromboembolism, atrial fibrillation, and acute coronary syndrome and those who had received orthopaedic surgery showed that the overall risk of gastrointestinal bleeding was $45 \%$ (95\% confidence interval $7 \%$ to $97 \%$ ) greater in patients treated with DOACs than in those who received standard care such as treatment with warfarin or low molecular weight heparin. ${ }^{34}$ Among venous thromboembolism patients only, treatment with DOACs increased the risk of gastrointestinal bleeding by $59 \%$ (3\% to $244 \%$ ) compared with standard care, although only two trials reported data on patients with venous thromboembolism. Our results show no increased risk of gastrointestinal bleeding associated with DOAC use.

Although our analysis of the safety of DOACs according to chronic kidney disease status showed similar bleeding risks across DOAC and warfarin groups, the safety profile of DOACs can vary by agent type depending on the percentage renal excretion. A systematic review assessing the safety of DOACs in patients with an estimated glomerular filtration rate of less than $50 \mathrm{~mL} / \mathrm{min}$ showed that the magnitude of relative reduction in major bleeding with DOAC use compared with warfarin was linearly associated with percentage renal excretion of the agent. ${ }^{36}$ The greatest relative reduction of major bleeding was observed for DOACs with renal excretion below 50\% (including rivaroxaban and apixaban), whereas those with renal excretion above 50\% (such as dabigatran) showed no reduction in the risk of major bleeding compared with warfarin.

\section{Strengths and limitations of study}

Our study formally pooled results from multiple healthcare settings, contributing to the generalisability of our findings. In addition, we assessed the safety of new DOAC and warfarin use, which removes the biases associated with safety assessments of prevalent drug use such as the underestimation of bleeding events.

Our study has some limitations. Despite the achieved balance in the included range of variables, owing to the observational nature of our study 


\begin{tabular}{|c|c|c|c|c|c|c|}
\hline \multirow[b]{2}{*}{ Centre } & \multicolumn{2}{|c|}{ No of events/total } & \multirow{2}{*}{\multicolumn{2}{|c|}{$\begin{array}{l}\text { Hazard ratio } \\
(95 \% \mathrm{Cl})\end{array}$}} & \multirow[b]{2}{*}{$\begin{array}{l}\text { Weight } \\
\text { (\%) }\end{array}$} & \multirow[b]{2}{*}{$\begin{array}{l}\text { Hazard ratio } \\
(95 \% \mathrm{Cl})\end{array}$} \\
\hline & DOAC & Warfarin & & & & \\
\hline \multicolumn{7}{|c|}{$\begin{array}{l}\text { Major bleeding } \\
\text { Chronic kidney disease }\end{array}$} \\
\hline Ontario & S/101 & $19 / 285$ & & & 19.3 & $0.72(0.27$ to 1.94$)$ \\
\hline Quebec & $12 / 100$ & $30 / 402$ & & & 42.2 & $1.80(0.93$ to 3.50$)$ \\
\hline MarketScan & $10 / 246$ & $38 / 853$ & & 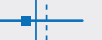 & 38.5 & 0.89 (0.44 to 1.78$)$ \\
\hline Overall & & & & & 100.0 & $1.15(0.75$ to 1.77$)$ \\
\hline \multicolumn{7}{|c|}{ Test for heterogeneity: $\chi^{2}=3.12, P=0.21, l^{2}=36 \%$} \\
\hline \multicolumn{7}{|c|}{ No chronic kidney disease } \\
\hline Alberta & $24 / 1456$ & $108 / 6345$ & & & 10.0 & $0.92(0.63$ to 1.34$)$ \\
\hline Ontario & $65 / 1980$ & $359 / 9597$ & & & 20.5 & $0.88(0.67$ to 1.14$)$ \\
\hline Quebec & $122 / 2444$ & $550 / 11977$ & & & 37.4 & 1.09 (0.90 to 1.33$)$ \\
\hline MarketScan & $113 / 5008$ & $358 / 13449$ & & ان & 32.1 & $0.84(0.68$ to 1.04$)$ \\
\hline Overall & & & & id & 100.0 & $0.94(0.84$ to 1.07$)$ \\
\hline \multicolumn{7}{|c|}{ Test for heterogeneity: $\chi^{2}=3.63, P=0.30, \mathrm{I}^{2}=17 \%$} \\
\hline \multicolumn{7}{|c|}{ All cause mortality } \\
\hline \multicolumn{7}{|c|}{ Chronic kidney disease } \\
\hline Ontario & $8 / 101$ & $34 / 285$ & & & 65.9 & $0.64(0.30$ to 1.38$)$ \\
\hline Quebec & $S / 100$ & $21 / 402$ & & & 34.1 & $0.76(0.26$ to 2.21$)$ \\
\hline Overall & & & & & 100.0 & $0.68(0.36$ to 1.27$)$ \\
\hline \multicolumn{7}{|c|}{ Test for heterogeneity: $\chi^{2}=0.06, P=0.80,1^{2}=0 \%$} \\
\hline \multicolumn{7}{|c|}{ No chronic kidney disease } \\
\hline Alberta & $33 / 1505$ & $147 / 6590$ & & 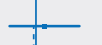 & 16.4 & $1.12(0.72$ to 1.74$)$ \\
\hline Ontario & $80 / 1980$ & $411 / 9597$ & & & 55.8 & 0.94 (0.74 to 1.20$)$ \\
\hline Quebec & $40 / 2444$ & 197/11 977 & & - & 27.8 & $1.00(0.71$ to 1.40$)$ \\
\hline Overall & & & & 1 & 100.0 & $0.98(0.82$ to 1.18$)$ \\
\hline \multicolumn{7}{|c|}{ Test for heterogeneity: $\chi^{2}=0.46, P=0.79, I^{2}=0 \%$} \\
\hline & & & 0.2 & 1 & 5 & \\
\hline & & & $\begin{array}{l}\text { Favours } \\
\text { DOAC }\end{array}$ & & & \\
\hline
\end{tabular}

Fig 4 | Hazard ratios (95\% Cls) of major bleeding and all cause mortality associated with DOAC use compared with warfarin use according to chronic kidney disease (CKD) status (reference category was warfarin users). $S=$ events were $<5$ and cells were suppressed. Some sites were not included in analysis owing to small number of DOAC users identified as having CKD (Manitoba and Saskatchewan) or small number of events observed across DOAC and warfarin groups (including zero events in DOAC group)

design, the possibility of residual confounding arising from differences in unmeasured variables remains. Although we assessed study outcomes within 90 and 180 days, the longer term safety of DOAC treatment for venous thromboembolism is less certain. Furthermore, most (95\%) DOAC users in our study used rivaroxaban, so we were unable to assess the safety of DOACs among venous thromboembolism patients by different DOAC agents. Future studies assessing the safety of other DOAC agents for the treatment of venous thromboembolism, including apixaban and dabigatran, are needed. Among warfarin users who had a major bleeding event, owing to the limited availability of international normalised ratio measurements in the study cohort, we were unable to determine the time within the therapeutic range or to adjust for over the counter use of aspirin or other nonsteroidal anti-inflammatory drugs.

Our algorithm for treated venous thromboembolism did not allow for an assessment according to deep venous thrombosis and pulmonary embolism status; nor could we differentiate distal from proximal venous thromboembolism or subsegmental from proximal pulmonary embolism. However, given the inclusion of oral anticoagulation as part of our venous thromboembolism algorithm, all events included in our study were associated with a treatment indication. Although the duration of DOAC or warfarin use was not assessed, given the short length of follow-up (90 days), the incident nature of the venous thromboembolism diagnosis, and the guideline recommended treatment of venous thromboembolism of at least 90 days, ${ }^{37}$ substantial misclassification of drug exposure is unlikely. The potential exists for misclassification of chronic kidney disease status based on ICD codes, but the high specificity of this algorithm $\left(99 \%{ }^{18}\right)$ would suggest that the small number of chronic kidney disease patients who may be misclassified would be minimal. Nonetheless, given the use of administrative codes, we were limited in our ability to ascertain whether a differential association between major bleeding risk and DOAC use exists according to level of kidney function. Additional studies are needed to further elucidate the safety of DOACs among venous thromboembolism patients with advanced chronic kidney disease. Although we used matching with replacement of warfarin users to maximise the number of DOAC users retained in the cohort, this results in a lack of independence among the warfarin users. However, we did sensitivity analyses that showed no effect on the precision of study findings. Finally, a random effects model for combining effect size estimates could have been selected instead of a fixed effects model. The former assumes that variation in effect size estimates can be attributed to both between study and within study variation. The selection of a random effects model can result in slightly larger confidence intervals and hence a more conservative test. However, the selection of a random effects model had no effect on the final conclusions as regards statistical significance.

\section{Conclusions}

In this analysis of data from large cohorts of adults with incident venous thromboembolism, treatment with DOACs, compared with warfarin, was not associated with an increased risk of major bleeding or all cause mortality in the first 90 days of treatment. These results were consistent for patients with and without chronic kidney disease, across all age groups, and for men and women.

\section{AUTHOR AFFILIATIONS}

${ }^{1}$ Departments of Medicine and Community Health Sciences, University of Calgary, AB, Canada

${ }^{2}$ The George Institute for Global Health, Sydney, NSW, Australia

${ }^{3}$ Faculty of Medicine, UNSW Sydney, NSW, Australia

${ }^{4}$ Department of Community Health Sciences, University of Manitoba, MB, Canada

${ }^{5}$ Department of Internal Medicine, University of Montreal Health Centre, Montreal, QC, Canada

${ }^{6}$ Manitoba Centre for Health Policy, Department of Community Health Sciences, University of Manitoba, Winnipeg, MB, Canada

${ }^{7}$ Department of Family Medicine, McMaster University, Hamilton, ON, Canada

${ }^{8}$ Institute for Clinical Evaluative Sciences, Toronto, ON, Canada 
${ }^{9}$ Institute of Health Policy, Management and Evaluation, University of Toronto, ON, Toronto

${ }^{10}$ Department of Anesthesiology, Pharmacology and Therapeutics, University of British Columbia, Vancouver, BC, Canada

${ }^{11}$ Center for Clinical Epidemiology, Lady Davis Research Institute, Jewish General Hospital, Montreal, QC, Canada

${ }^{12}$ Department of Medicine, McGill University, Montreal, QC, Canada

${ }^{13}$ College of Pharmacy and Nutrition, Department of Pharmacy,

University of Saskatchewan, SK, Canada

${ }^{14}$ Department of Neurology and Neurosurgery, McGill University, Montréal, QC, Canada

${ }^{15}$ Department of Epidemiology and Biostatistics, McGill University, Montréal, QC, Canada

${ }^{16}$ School of Kinesiology and Health Science, York University, Toronto, ON, Canada

${ }^{17}$ Department of Community Health and Epidemiology, Dalhousie University, Halifax, NS, Canada

${ }^{18}$ Department of Medicine, University of Alberta, Edmonton, AB, Canada

${ }^{19}$ Department of Community Health Sciences, University of Manitoba, Winnipeg, MB, Canada

Canadian Network for Observational Drug Effect Studies (CNODES) Investigators: Samy Suissa (principal investigator); Colin R Dormuth (British Columbia); Brenda R Hemmelgarn (Alberta); Gary F Teare (Saskatchewan); Patricia Caetano and Dan Chateau (Manitoba); David A Henry and I Michael Paterson (Ontario); Jacques LeLorier (Québec); Adrian R Levy (Atlantic: NS, NL, NB, PEI); Pierre Ernst and Kristian B Filion (UK CPRD); Robert W Platt (methods); and Ingrid S Sketris (knowledge translation).

We acknowledge the contributions of Alexis Matteau (Quebec) and the programming support of Zhihai Ma and Jianguo Zhang (Alberta), Sophie Dell'Aniello (CPRD), Yan Wang (Nova Scotia), Caixia Fangyun Wu (Ontario), Thierry Ducruet (Quebec), Nianping Hu (Saskatchewan), and Gregory A Carney (US MarketScan). We also acknowledge the important contributions of the CNODES collaborators and assistants at each site. This study was made possible through data sharing agreements between CNODES member research centres and the respective provincial governments of Alberta, Manitoba (HIPC \# 2015/2016 - 47), Nova Scotia, Ontario, Quebec, and Saskatchewan. The opinions, results, and conclusions reported in this paper are those of the authors. No endorsement by the provinces is intended or should be inferred. Parts of this material are based on data and information compiled and provided by the Canadian Institute for Health Information (CIHI). However, the analyses, conclusions, opinions, and statements expressed herein are those of the authors and not necessarily those of $\mathrm{ClHI}$.

Contributors: MJ, LML, MDurand, MDahl, MJP, CRD, PE, SY, HT, SMM, and BRH were responsible for study concept and design; data collection, analysis, and interpretation; and manuscript preparation. $\mathrm{MJ}$ and BRH drafted the initial manuscript, and all authors contributed to data interpretation and critical revision of the report. BRH is the guarantor.

Funding: MJ was supported by postdoctoral fellowships from the Canadian Institutes of Health Research (CIHR) and Alberta Innovates Health Solutions (AIHS) and an early career fellowship from the National Health and Medical Research Council of Australia (NHMRC). MDurand is supported by a salary award from the Fonds de Recherche du Québec-Santé. BRH was supported by the Roy and Vi Baay Chair in Kidney Research. LML was supported by a Manitoba Research Chair. CNODES, a collaborating centre of the Drug Safety and Effectiveness Network (DSEN), is funded by the CIHR (grant number DSE-146021). The funding organisations had no role in the design and conduct of the study; in the collection, analysis, and interpretation of the data; or in the preparation, review, or approval of the manuscript.

Competing interests: All authors have completed the ICMJE uniform disclosure form at www.icmje.org/coi disclosure.pdf (available on request from the corresponding author) and declare: support for the submitted work as described above; CW has received honorariums (for advisory board meetings as well as speaking engagements) from Leo Pharma and Pfizer (makers of tinzaparin and dalteparin, respectively); SMM has received research grants for work unrelated to this project from GlaxoSmithKline, Merck, Pfizer, and Sanofi; no other relationships or activities that could appear to have influenced the submitted work.
Ethical approval: The study protocol was approved by the institutional review boards at all participating sites and by the Independent Scientific Advisory Committee (ISAC; protocol number 16_110R) of the CPRD. The study protocol is registered at www. clinicaltrials.gov (NCT02833987). The data are anonymous, and the requirement for informed consent was therefore waived.

Data sharing: No additional data available.

Transparency: The lead author affirms that the manuscript is an honest, accurate and transparent account of the study being reported; that no important aspects of the study have been omitted; and that any discrepancies from the study as planned (and, if relevant, registered) have been explained.

This is an Open Access article distributed in accordance with the Creative Commons Attribution Non Commercial (CC BY-NC 4.0) license, which permits others to distribute, remix, adapt, build upon this work non-commercially, and license their derivative works on different terms, provided the original work is properly cited and the use is noncommercial. See: http://creativecommons.org/licenses/by-nc/4.0/.

1 White RH. The epidemiology of venous thromboembolism. Circulation 2003;107(Suppl 1):14-8. doi:10.1161/01. CIR.0000078468.11849.66

2 Kahn SR, Morrison DR, Cohen JM, et al. Interventions for implementation of thromboprophylaxis in hospitalized medical and surgical patients at risk for venous thromboembolism. Cochrane Database Syst Rev 2013;7:CD008201.

3 Hawkins D. Limitations of traditional anticoagulants. Pharmacotherapy 2004:24:62S-5S. doi:10.1592/ phco.24.10.62S.36120

4 Gonsalves WI, Pruthi RK, Patnaik MM. The new oral anticoagulants in clinical practice. Mayo Clin Proc 2013;88:495-511. doi:10.1016/ j.mayocp.2013.03.006

5 Agnelli G, Buller HR, Cohen A, et al. AMPLIFY Investigators, Oral apixaban for the treatment of acute venous thromboembolism. N Engl J Med 2013;369:799-808. doi:10.1056/NEJMoa1302507

6 Bauersachs R, Berkowitz SD, Brenner B, et al. EINSTEIN Investigators. Oral rivaroxaban for symptomatic venous thromboembolism. N Engl J Med 2010;363:2499-510. doi:10.1056/NEJMoa1007903

7 Schulman S, Kearon C, Kakkar AK, et al. RE-COVER Study Group. Dabigatran versus warfarin in the treatment of acute venous thromboembolism. N Engl/ Med 2009;361:2342-52. doi:10.1056/ NEJMoa0906598

8 Büller HR, Prins MH, Lensin AW, et al. EINSTEIN-PE Investigators. Ora rivaroxaban for the treatment of symptomatic pulmonary embolism. N Engl J Med 2012;366:1287-97. doi:10.1056/NEJMoa1113572

9 Wang SV, Franklin JM, Glynn RJ, Schneeweiss S, Eddings W, Gagne II. Prediction of rates of thromboembolic and major bleeding outcomes with dabigatran or warfarin among patients with atrial fibrillation: new initiator cohort study. BMJ 2016;353:i2607. doi:10.1136/ bmj.i2607

10 Alotaibi GS, Wu C, Senthilselvan A, McMurtry MS. The validity of ICD codes coupled with imaging procedure codes for identifying acute venous thromboembolism using administrative data. Vasc Med 2015:20:364-8 doi:10.1177/1358863X15573839

11 Sanfilippo KM, Wang TF, Gage BF, Liu W, Carson KR. Improving accuracy of International Classification of Diseases codes for venous thromboembolism in administrative data. Thromb Res 2015;135:616-20. doi:10.1016/j.thromres.2015.01.012

12 Tamariz L, Harkins T, Nair V. A systematic review of validated methods for identifying venous thromboembolism using administrative and claims data. Pharmacoepidemiol Drug Saf 2012;21(Suppl 1): 154-62. doi:10.1002/pds.2341

13 Arnason T, Wells PS, van Walraven C, Forster Al. Accuracy of coding for possible warfarin complications in hospital discharge abstracts. Thromb Res 2006;118:253-62. doi:10.1016/j. thromres.2005.06.015

14 Gomes T, Mamdani MM, Holbrook AM, Paterson IM, Hellings C Juurlink DN. Rates of hemorrhage during warfarin therapy for atrial fibrillation. CMAJ 2013;185:E121-7. doi:10.1503/cmaj.121218

15 Jun M, James MT, Ma Z, et al. Alberta Kidney Disease Network. Warfarin initiation, atrial fibrillation, and kidney function: comparative effectiveness and safety of warfarin in older adults with newly diagnosed atrial fibrillation. Am J Kidney Dis 2017;69:734-43. doi:10.1053/i.ajkd.2016.10.018

16 Austin PC. An introduction to propensity score methods for reducing the effects of confounding in observational studies. Multivariate Behav Res 2011:46:399-424. doi:10.1080/00273171.2011. 568786

17 Hougaard P. Analysis of multivariate survival data. Springer, 2000doi:10.1007/978-1-4612-1304-8.

18 Fleet JL, Dixon SN, Shariff SZ, et al. Detecting chronic kidney disease in population-based administrative databases using an algorithm of hospital encounter and physician claim codes. BMC Nephrol 2013:14:81. doi:10.1186/1471-2369-14-81 
19 Ronksley PE, Tonelli M, Quan H, et al. Alberta Kidney Disease Network. Validating a case definition for chronic kidney disease using administrative data. Nephrol Dial Transplant 2012;27:1826-31. doi:10.1093/ndt/gfr598

20 Vlasschaert ME, Bejaimal SA, Hackam DG, et al. Validity of administrative database coding for kidney disease: a systematic review. Am J Kidney Dis 2011;57:29-43. doi:10.1053/j. ajkd.2010.08.031

21 Martinez C, Cohen AT, Bamber L, Rietbrock S. Epidemiology of first and recurrent venous thromboembolism: a populationbased cohort study in patients without active cancer. Thromb Haemost 2014;112:255-63. doi:10.1160/TH13-09-0793

22 Gallagher AM, Williams T, Leufkens HG, de Vries F. The impact of the choice of data source in record linkage studies estimating mortality in venous thromboembolism. PLoS One 2016;11:e0148349. doi:10.1371/journal.pone.0148349

23 van der Hulle T, Kooiman J, den Exter PL, Dekkers OM, Klok FA, Huisman MV. Effectiveness and safety of novel oral anticoagulants as compared with vitamin $\mathrm{K}$ antagonists in the treatment of acute symptomatic venous thromboembolism: a systematic review and meta-analysis. J Thromb Haemost 2014;12:320-8. doi:10.1111/ jth.12485

24 Sterne JA, Bodalia PN, Bryden PA, et al. Oral anticoagulants for primary prevention, treatment and secondary prevention of venous thromboembolic disease, and for prevention of stroke in atrial fibrillation: systematic review, network meta-analysis and cost-effectiveness analysis. Health Technol Assess 2017;21:1-386. doi: $10.3310 /$ hta21090

25 Gaertner S, Cordeanu EM, Nouri S, et al. Rivaroxaban versus standard anticoagulation for symptomatic venous thromboembolism (REMOTEV observational study): Analysis of 6-month outcomes. Int) Cardiol 2017;226:103-9. doi:10.1016/j.ijcard.2016.10.045

26 Keller L, Marten S, Hecker J, Werth S, Tittl L, Beyer-Westendorf J. Treatment of acute VTE with rivaroxaban - results of the prospective Dresden NOAC Registry (NCT01588119). Blood 2016;128:2618.

27 Kucher N, Aujesky D, Beer H et al. Rivaroxaban for the treatment of venous thromboembolism. The SWIss Venous ThromboEmbolism Registry (SWIVTER). Thromb Haemost 2016;116:472-9. doi:10.1160/TH16-03-0209
28 Larsen TB, Skjøth F, Kjældgaard JN, Lip GYH, Nielsen PB, Søgaard M. Effectiveness and safety of rivaroxaban and warfarin in patients with unprovoked venous thromboembolism: a propensity-matched nationwide cohort study. Lancet Haematol 2017;4:e237-44. doi:10.1016/S2352-3026(17)30054-6

29 Peacock W, Tamayo S, Patel M, Sicignano N, Hopf K, Yuan Z. Major bleeding in patients taking rivaroxaban for VTE treatment. Chest 2016;150:286Adoi:10.1016/j.chest.2016.08.299.

30 Ageno W, Mantovani LG, Haas S, et al. Safety and effectiveness of oral rivaroxaban versus standard anticoagulation for the treatment of symptomatic deep-vein thrombosis (XALIA): an international, prospective, non-interventional study. Lancet Haematol 2016;3: 12-21. doi:10.1016/S2352-3026(15)00257-4

31 Wang L, Baser O, Wells P, et al. Effectiveness of rivaroxaban among patients diagnosed with pulmonary embolism. Circulation 2016:134:A13140.

32 Sindet-Pedersen C, Langtved Pallisgaard J, Staerk L, et al. Comparative safety and effectiveness of rivaroxaban versus VKAs in patients with venous thromboembolism. A Danish nationwide registry-based study. Thromb Haemost 2017;117:1182-91. doi:10.1160/TH16-10-0745

33 Connolly SJ, Ezekowitz MD, Yusuf S, et al. RE-LY Steering Committee and Investigators. Dabigatran versus warfarin in patients with atrial fibrillation. N Engl J Med 2009;361:1139-51. doi:10.1056/ NEJMoa0905561

34 Holster IL, Valkhoff VE, Kuipers EJ, Tjwa ET. New oral anticoagulants increase risk for gastrointestinal bleeding: a systematic review and meta-analysis. Gastroenterology 2013;145:105-112.e15. doi:10.1053/j.gastro.2013.02.041

35 Patel MR, Mahaffey KW, Garg J, et al. ROCKET AF Investigators. Rivaroxaban versus warfarin in nonvalvular atrial fibrillation. N Engl) Med 2011;365:883-91. doi:10.1056/NEJMoa1009638

36 Lega IC, Bertoletti L, Gremillet C, Boissier C, Mismetti P, Laporte S. Consistency of safety profile of new oral anticoagulants in patients with renal failure. J Thromb Haemost 2014;12:337-43. doi:10.1111/ jth.12486

37 Kearon C, Akl EA, Ornelas J, et al. Antithrombotic therapy for VTE disease: CHEST guideline and expert panel report. Chest 2016;149:315-52. doi:10.1016/j.chest.2015.11.026 